\title{
THE WEAK JOB RECOVERY IN A MACRO MODEL OF SEARCH AND RECRUITING INTENSITY (ONLINE APPENDIX)
}

\author{
SYLVAIN LEDUC AND ZHENG LIU
}

\begin{abstract}
This appendix provides a summary of equilibrium conditions in the DSGE model of Leduc and Liu (2019) and presents some additional results with alternative assumptions on the entry-cost distribution.
\end{abstract}

\section{Summary of equilibrium Conditions in the DSGE MOdel}

A search equilibrium is a system of 18 equations for 18 variables summarized in the vector

$$
\left[C_{t}, \Lambda_{t}, m_{t}, q_{t}^{u}, q_{t}^{v}, N_{t}, u_{t}, U_{t}, Y_{t}, r_{t}, v_{t}, J_{t}^{F}, w_{t}^{N}, w_{t}, n_{t}, a_{t}, s_{t}, J_{t}^{V}\right] .
$$

We write the equations in the same order as in the dynare code.

(1) Household's bond Euler equation:

$$
1=\mathrm{E}_{t} \beta \theta_{t+1} \frac{\Lambda_{t+1}}{\Lambda_{t}} r_{t}
$$

(2) Marginal utility of consumption

$$
\Lambda_{t}=\frac{1}{C_{t}}
$$

(3) Search intensity

$$
\begin{aligned}
h_{1} & +h_{2}\left(s_{t}-\bar{s}\right) \\
& =\frac{q_{t}^{u}}{s_{t}}\left[w_{t}-\phi-\frac{\chi}{\Lambda_{t}}+\mathrm{E}_{t} \frac{\beta \theta_{t+1} \Lambda_{t+1}}{\Lambda_{t}}\left(1-\rho^{o}\right)\left(1-\delta_{t+1}\right)\left(1-q_{t+1}^{u}\right) \frac{b}{1-b}\left(J_{t+1}^{F}-J_{t+1}^{V}\right)\right] \\
& =\frac{q_{t}^{u}}{s_{t}}\left[w_{t}-w_{t}^{N}-\frac{h\left(s_{t}\right)}{1-q_{t}^{u}}+\frac{b}{1-b}\left(J_{t}^{F}-J_{t}^{V}\right)\right],
\end{aligned}
$$

where the second equality follows from the Nash bargaining condition (B16).

Date: February 6, 2019.

Leduc: Federal Reserve Bank of San Francisco, 101 Market Street, San Francisco, CA 94105 (email: Sylvain.Leduc@sf.frb.org). Liu: Federal Reserve Bank of San Francisco, 101 Market Street, San Francisco, CA 94105 (email: Zheng.Liu@sf.frb.org). The views expressed herein are those of the authors and do not necessarily reflect the views of the Federal Reserve Bank of San Francisco or of the Federal Reserve System. 
(4) Matching function

$$
m_{t}=\mu_{t}\left(s_{t} u_{t}\right)^{\alpha}\left(a_{t} v_{t}\right)^{1-\alpha}
$$

(5) Job finding rate

$$
q_{t}^{u}=\frac{m_{t}}{u_{t}}
$$

(6) Vacancy filling rate

$$
q_{t}^{v}=\frac{m_{t}}{v_{t}}
$$

(7) Employment dynamics:

$$
N_{t}=\left(1-\rho^{o}\right)\left(1-\delta_{t}\right) N_{t-1}+m_{t} \text {, }
$$

(8) Number of searching workers:

$$
u_{t}=1-\left(1-\rho^{o}\right)\left(1-\delta_{t}\right) N_{t-1},
$$

(9) Unemployment:

$$
U_{t}=1-N_{t},
$$

(10) Law of motion for vacancies:

$$
v_{t}=\left(1-\rho^{o}\right)\left(1-q_{t-1}^{v}\right) v_{t-1}+\delta_{t}\left(1-\rho^{o}\right) N_{t-1}+n_{t},
$$

(11) Aggregate production function:

$$
Y_{t}=Z_{t} N_{t}
$$

(12) Aggregate Resource constraint:

$$
C_{t}+h\left(s_{t}\right) u_{t}+\kappa\left(a_{t}\right) v_{t}+\frac{\xi}{1+\xi} n_{t} J_{t}^{V}=Y_{t},
$$

where the search cost function and the recruiting cost function are given by

$$
\begin{aligned}
h\left(s_{t}\right) & =h_{1}\left(s_{t}-\bar{s}\right)+\frac{h_{2}}{2}\left(s_{t}-\bar{s}\right)^{2} \\
\kappa\left(a_{t}\right) & =\kappa_{0}+\kappa_{1}\left(a_{t}-\bar{a}\right)+\frac{\kappa_{2}}{2}\left(a_{t}-\bar{a}\right)^{2}
\end{aligned}
$$

(13) Value of vacancy:

$$
J_{t}^{V}=-\kappa\left(a_{t}\right)+q_{t}^{v} J_{t}^{F}+\left(1-q_{t}^{v}\right)\left(1-\rho^{o}\right) E_{t} \frac{\beta \theta_{t+1} \Lambda_{t+1}}{\Lambda_{t}} J_{t+1}^{V},
$$

(14) Recruiting intensity:

$$
\kappa_{1}+\kappa_{2}\left(a_{t}-\bar{a}\right)=\frac{q_{t}^{v}}{a_{t}}\left[J_{t}^{F}-\left(1-\rho^{o}\right) E_{t} \frac{\beta \theta_{t+1} \Lambda_{t+1}}{\Lambda_{t}} J_{t+1}^{V}\right] .
$$

(15) Match value:

$$
J_{t}^{F}=Z_{t}-w_{t}+E_{t} \frac{\beta \theta_{t+1} \Lambda_{t+1}}{\Lambda_{t}}\left(1-\rho^{o}\right)\left\{\left(1-\delta_{t+1}\right) J_{t+1}^{F}+\delta_{t+1} J_{t+1}^{V}\right\}
$$


(16) Nash bargaining wage:

$\frac{b}{1-b}\left(J_{t}^{F}-J_{t}^{V}\right)=w_{t}^{N}-\phi-\frac{\chi_{t}}{\Lambda_{t}}+\frac{h\left(s_{t}\right)}{1-q_{t}^{u}}+E_{t} \frac{\beta \theta_{t+1} \Lambda_{t+1}}{\Lambda_{t}}\left(1-\rho^{o}\right)\left(1-\delta_{t+1}\right)\left(1-q_{t+1}^{u}\right) \frac{b}{1-b}\left(J_{t+1}^{F}-J_{t+1}^{V}\right)$.

(17) Actual real wage (with real wage rigidity)

$$
w_{t}=w_{t-1}^{\gamma}\left(w_{t}^{N}\right)^{1-\gamma}
$$

(18) New vacancy

$$
K n_{t}=\left(J_{t}^{V}\right)^{\xi}
$$

\section{Steady State}

(1) Household's bond Euler equation:

$$
1=\beta r
$$

(2) Marginal utility of consumption

$$
\Lambda=\frac{1}{C}
$$

(3) Search intensity

$$
h_{1}=\frac{q^{u}}{s}\left[w-\phi-\frac{\chi}{\Lambda}+\beta\left(1-\rho^{o}\right)(1-\delta)\left(1-q^{u}\right) \frac{b}{1-b}\left(J^{F}-J^{V}\right)\right],
$$

(4) Matching function

$$
m=\mu(\bar{s} u)^{\alpha}(\bar{a} v)^{1-\alpha},
$$

(5) Job finding rate

$$
q^{u}=\frac{m}{u}
$$

(6) Vacancy filling rate

$$
q^{v}=\frac{m}{v}
$$

(7) Employment dynamics:

$$
m=\left[\delta+(1-\delta) \rho^{o}\right] N,
$$

(8) Number of searching workers:

$$
u=U+m,
$$

(9) Unemployment:

$$
U=1-N
$$

(10) Vacancies:

$$
\left[q^{v}+\rho^{o}\left(1-q^{v}\right)\right] v=\delta\left(1-\rho^{o}\right) N+n,
$$


(11) Aggregate production function:

$$
Y=Z N
$$

(12) Aggregate Resource constraint:

$$
C+\kappa_{0} v+\frac{\xi}{1+\xi} n J^{V}=Y,
$$

(13) Value of vacancies:

$$
q^{v} J^{F}-\kappa_{0}=\left[1-\beta\left(1-q^{v}\right)\left(1-\rho^{o}\right)\right] J^{V}
$$

(14) Recruiting intensity:

$$
\kappa_{1} \bar{a}=q^{v}\left[J^{F}-\beta\left(1-\rho^{o}\right) J^{V}\right]
$$

(15) Match value:

$$
\left[1-\beta(1-\delta)\left(1-\rho^{o}\right)\right] J^{F}=Z-w+\beta\left(1-\rho^{o}\right) \delta J^{V},
$$

(16) Nash bargaining wage:

$$
w^{N}=\phi+\frac{\chi}{\Lambda}+\frac{b}{1-b}\left[1-\beta\left(1-\rho^{o}\right)(1-\delta)\left(1-q^{u}\right)\right]\left(J^{F}-J^{V}\right),
$$

(17) Actual real wage

$$
w=w^{N},
$$

(18) New vacancy

$$
K n=\left(J^{V}\right)^{\xi}
$$

III. Equilibrium System SCAled by Steady state (USEd in dynare)

Denote by $\hat{X}_{t} \equiv \frac{X_{t}}{X}$ the scaled value of the variable $X_{t}$ by its steady-state level. The system of equilibrium conditions can be reduced to the following 18 equations to solve for the 18 endogenous variables summarized in the vector

$$
\left[\hat{C}_{t}, \hat{\Lambda}_{t}, \hat{r}_{t}, \hat{Y}_{t}, \hat{m}_{t}, \hat{u}_{t}, \hat{v}_{t}, \hat{q}_{t}^{u}, \hat{q}_{t}^{v}, \hat{N}_{t}, \hat{U}_{t}, \hat{J}_{t}^{F}, \hat{w}_{t}^{N}, \hat{w}_{t}, \hat{n}_{t}, \hat{a}_{t}, \hat{s}_{t}, \hat{J}_{t}^{V}\right]
$$

(1) Household's bond Euler equation:

$$
1=\mathrm{E}_{t} \exp \left(\hat{\theta}_{t+1}\right) \frac{\hat{\Lambda}_{t+1}}{\hat{\Lambda}_{t}} \hat{r}_{t}
$$

(2) Marginal utility of consumption

$$
\hat{\Lambda}_{t}=\frac{1}{\hat{C}_{t}},
$$


(3) Search intensity

$$
h_{1}+h_{2} \bar{s}\left(\hat{s}_{t}-1\right)=\frac{q^{u} \hat{q}_{t}^{u}}{\bar{s} s_{t}}\left\{\frac{b}{1-b}\left(J^{F} \hat{J}_{t}^{F}-J^{V} \hat{J}_{t}^{V}\right)+w\left(\hat{w}_{t}-\hat{w}_{t}^{N}\right)-\frac{h_{1} \bar{s}\left(\hat{s}_{t}-1\right)+\frac{h_{2} \bar{s}^{2}}{2}\left(\hat{s}_{t}-1\right)^{2}}{1-q^{u} \hat{q}_{t}^{u}}\right\}
$$

(4) Matching function

$$
\hat{m}_{t}=\left(\hat{s}_{t} \hat{u}_{t}\right)^{\alpha}\left(\hat{a}_{t} \hat{v}_{t}\right)^{1-\alpha},
$$

(5) Job finding rate

$$
\hat{q}_{t}^{u}=\frac{\hat{m}_{t}}{\hat{u}_{t}}
$$

(6) Vacancy filling rate

$$
\hat{q}_{t}^{v}=\frac{\hat{m}_{t}}{\hat{v}_{t}}
$$

(7) Employment dynamics:

$$
\hat{N}_{t}=\left(1-\rho^{o}\right)\left(1-\delta \exp \left(\hat{\delta}_{t}\right)\right) \hat{N}_{t-1}+\frac{m}{N} \hat{m}_{t},
$$

(8) Number of searching workers

$$
u \hat{u}_{t}=1-\left(1-\rho^{o}\right)\left(1-\delta \exp \left(\hat{\delta}_{t}\right)\right) N \hat{N}_{t-1},
$$

(9) Unemployment:

$$
U \hat{U}_{t}=1-N \hat{N}_{t}
$$

(10) Vacancies:

$$
v \hat{v}_{t}=\left(1-\rho^{o}\right)\left(1-q^{v} \hat{q}_{t-1}^{v}\right) v \hat{v}_{t-1}+\delta \exp \left(\hat{\delta}_{t}\right)\left(1-\rho^{o}\right) N \hat{N}_{t-1}+n \hat{n}_{t},
$$

(11) Aggregate production function:

$$
\hat{Y}_{t}=\exp \left(\hat{z}_{t}\right) \hat{N}_{t}
$$

(12) Aggregate Resource constraint:

$$
\begin{aligned}
\hat{Y}_{t} & =\left[h_{1} \bar{s}\left(\hat{s}_{t}-1\right)+\frac{h_{2} \bar{s}^{2}}{2}\left(\hat{s}_{t}-1\right)^{2}\right] \frac{u}{Y} \hat{u}_{t}+\left[\kappa_{0}+\kappa_{1} \bar{a}\left(\hat{a}_{t}-1\right)+\frac{\kappa_{2} \bar{a}^{2}}{2}\left(\hat{a}_{t}-1\right)^{2}\right] \frac{v}{Y} \hat{v}_{t} \\
& +\frac{C}{Y} \hat{C}_{t}+\frac{\xi}{1+\xi} \frac{n J^{V}}{Y}\left(\hat{n}_{t}+\hat{J}_{t}^{V}\right)
\end{aligned}
$$

(13) Value of vacancy:

$$
\begin{gathered}
J^{V} \hat{J}_{t}^{V}=-\left[\kappa_{0}+\kappa_{1} \bar{a}\left(\hat{a}_{t}-1\right)+\frac{\kappa_{2} \bar{a}^{2}}{2}\left(\hat{a}_{t}-1\right)^{2}\right]+ \\
q^{v} J^{F} \hat{q}_{t}^{v} \hat{J}_{t}^{F}+\left(1-q^{v} \hat{q}_{t}^{v}\right)\left(1-\rho^{o}\right) \mathrm{E}_{t} \frac{\beta \exp \left(\hat{\theta}_{t+1}\right) \hat{\Lambda}_{t+1}}{\hat{\Lambda}_{t}} J^{V} \hat{J}_{t+1}^{V}
\end{gathered}
$$


(14) Recruiting intensity:

$$
\kappa_{1}+\kappa_{2} \bar{a}\left(\hat{a}_{t}-1\right)=\frac{q^{v} \hat{q}_{t}^{v}}{\bar{a} \hat{a}_{t}}\left[J^{F} \hat{J}_{t}^{F}-\left(1-\rho^{o}\right) \mathrm{E}_{t} \frac{\beta \exp \left(\hat{\theta}_{t+1}\right) \hat{\Lambda}_{t+1}}{\hat{\Lambda}_{t}} J^{V} \hat{J}_{t+1}^{V}\right] .
$$

(15) Match value:

$J^{F} \hat{J}_{t}^{F}=\exp \left(\hat{z}_{t}\right)-w \hat{w}_{t}+\mathrm{E}_{t} \frac{\beta \exp \left(\hat{\theta}_{t+1}\right) \hat{\Lambda}_{t+1}}{\hat{\Lambda}_{t}}\left(1-\rho^{o}\right)\left\{\left(1-\delta \exp \left(\hat{\delta}_{t+1}\right)\right) J^{F} \hat{J}_{t+1}^{F}+\delta \exp \left(\hat{\delta}_{t+1}\right) J^{V} \hat{J}_{t+1}^{V}\right\}$,

(16) Nash bargaining wage:

$$
\begin{aligned}
& \frac{b}{1-b}\left(J^{F} \hat{J}_{t}^{F}-J^{V} \hat{J}_{t}^{V}\right)=w \hat{w}_{t}^{N}-\phi-\frac{\chi}{\Lambda \hat{\Lambda}_{t}}+\frac{h_{1} \bar{s}\left(\hat{s}_{t}-1\right)+\frac{h_{2} \bar{s}^{2}}{2}\left(\hat{s}_{t}-1\right)^{2}}{1-q^{u} \hat{q}_{t}^{u}}+ \\
& E_{t} \frac{\beta \exp \left(\hat{\theta}_{t+1}\right) \hat{\Lambda}_{t+1}}{\hat{\Lambda}_{t}}\left(1-\rho^{o}\right)\left[\left(1-\delta \exp \left(\hat{\delta}_{t+1}\right)\right)\left(1-q^{u} \hat{q}_{t+1}^{u}\right) \frac{b}{1-b}\left(J^{F} \hat{J}_{t+1}^{F}-J^{V} \hat{J}_{t+1}^{V}(0)\right] 16\right)
\end{aligned}
$$

(17) Actual real wage (with real wage rigidity)

$$
\hat{w}_{t}=\hat{w}_{t-1}^{\gamma}\left(\hat{w}_{t}^{N}\right)^{1-\gamma}
$$

(18) New vacancies

$$
\hat{n}_{t}=\xi \hat{J}_{t}^{V}
$$

(19) Technology shock process

$$
\hat{z}_{t}=\rho_{z} \hat{z}_{t-1}+\varepsilon_{z t}
$$

(20) Discount factor shock process

$$
\hat{\theta}_{t}=\rho_{\theta} \hat{\theta}_{t-1}+\varepsilon_{\theta t},
$$

(21) Job separation shock process

$$
\hat{\delta}_{t}=\rho_{\delta} \hat{\delta}_{t-1}+\varepsilon_{\delta t},
$$

\section{HistoricAl DECOMPOSITION}

Figure A1 shows the historical decompositions of the shocks that drive the fluctuations in the unemployment rate from our benchmark estimation. Consistent with the forecast error variance decomposition discussed in the text, the unemployment fluctuations are driven mostly by the discount factor shock and the technology shock, and the job separation shock plays a relative minor role. The figure also shows that the surge in the unemployment from 2007 to 2009 was driven primarily by the discount factor shock, which captures financial frictions not in our model. During the recovery period from 2010 and onwards, technology shocks were more important for the unemployment dynamics. 
Figure A2 shows the historical decomposition for the fluctuations in job vacancies. As in the case for the unemployment rate, the sharp declines in vacancies in the Great Recession were mainly driven by discount factor shocks, and technology shocks played a more important role during the recovery.

Figure A3 displays the historical decomposition results for search intensity fluctuations. Again, discount factor shocks were the driving force for the sharp downturn during the recession, and technology shocks played a more important role during the recovery.

For all three series, job separation shocks are not an important driving factor.

\section{Posterior PREDiCTIVE CHECKS}

Table A1 displays the posterior predictive checks for the estimation results when we calibrate the stochastic processes of the technology shock and the job separation shock following Shimer (2005). The calibrated persistence parameters are shown in the first column of Panel A, and the calibrated variances of the two shocks are shown in the first column of Panel B. As we discuss in the text (in Section V.3), we examine the extent to which the quantitative results from our benchmark estimation are driven by the estimated shocks versus the model's internal propagation mechanism, we estimate the model using an alternative approach. In particular, we first fix the parameters in the technology shock and the separation shock at their calibrated values and then estimate the structural parameters along with the discount factor process to fit the model to the time series of unemployment, vacancies, and search intensity. We have discussed the estimation results in the text (and Table 4 in the paper). We have also noted that the data prefer the benchmark estimation to this alternative approach because the posterior data density under the benchmark estimation is much higher.

To check whether the actual data lie in the tail of the model's posterior distribution when we restrict the stochastic processes of the technology shock and the separation shock in our estimation, we perform a posterior predictive check following the approach described by An and Schorfheide (2007) and Faust and Gupta (2012). The second column of Table A1 shows the $90 \%$ posterior range for the calibrated shock parameters based on 2000 draws from the posterior smoothed series of productivity and the job separation rate. The calibrated values lie within the $90 \%$ posterior range. The third column shows the posterior median values. The calibrated shock parameters all lie within the $90 \%$ posterior range and not far from the posterior median.

\section{Estimation RESUlts Without USing SEARCH INTENSITy DATA}

Table A2 shows the estimated parameters in the case where we estimate the model to fit the time series of unemployment and vacancies, without using information from search 
intensity. Compared to the benchmark estimation, this alternative estimation without using information from search intensity data results in a few notable changes in the posterior distributions of the parameters. In particular, the technology shock becomes much less persistent than in the benchmark (with a persistence of 0.185 here vs. 0.995 in the benchmark). The standard deviation of the discount factor shock is smaller than in the benchmark (0.0076 vs. 0.0302). The separation shock parameters are similar to those in the benchmark. 
TABLE A1. Posterior predictive checks for alternative estimation

\begin{tabular}{llll}
\hline \hline \multicolumn{4}{l}{ A. First-order autocorrelations } \\
\hline Shocks & Calibrated & Posterior 90\% range & Posterior median \\
\hline Technology shock & 0.9908 & {$[0.9585,0.9913]$} & 0.9814 \\
Job separation shock & 0.9806 & {$[0.9478,0.9849]$} & 0.9724 \\
\hline \multicolumn{4}{l}{ B. Variances } \\
\hline Shocks & Calibrated & Posterior 90\% range & Posterior median \\
\hline Technology shock & 0.0013 & {$[0.0003,0.0018]$} & 0.0007 \\
Job separation shock & 0.0001 & {$[0.0000,0.0002]$} & 0.0001 \\
\hline \hline
\end{tabular}

Note: The $90 \%$ ranges and the medians of these shock parameters are calculated based on 2000 draws from the posterior smoothed series of the two shocks, following the approach of An and Schorfheide (2007) for posterior predictive checks. 
TABle A2. Estimated parameters

\begin{tabular}{|c|c|c|c|c|c|c|}
\hline & \multirow[b]{2}{*}{ Parameter description } & \multicolumn{2}{|c|}{ Priors } & \multicolumn{3}{|c|}{ Posterior } \\
\hline & & Type & {$[$ mean, std $]$} & Mean & $5 \%$ & $95 \%$ \\
\hline$K$ & vacancy creation cost & $\mathrm{G}$ & {$[5,1]$} & 5.0875 & 3.6425 & 6.5918 \\
\hline$\kappa_{2}$ & vacancy posting cost & G & {$[5,1]$} & 2.9834 & 1.3555 & 4.2564 \\
\hline$h_{2}$ & search cost function & G & {$[5,1]$} & 5.4664 & 3.1973 & 7.2110 \\
\hline$\rho_{z}$ & $\mathrm{AR}(1)$ of tech shock & $\mathrm{B}$ & {$[0.8,0.1]$} & 0.1848 & 0.1318 & 0.2357 \\
\hline$\sigma_{z}$ & std of tech shock & $\mathrm{IG}$ & {$[0.01,0.1]$} & 0.0123 & 0.0108 & 0.0135 \\
\hline$\rho_{\theta}$ & $\mathrm{AR}(1)$ of dis. factor shock & $\mathrm{B}$ & {$[0.8,0.1]$} & 0.9907 & 0.9866 & 0.9962 \\
\hline$\sigma_{\theta}$ & std of dis. factor shock & IG & {$[0.01,0.1]$} & 0.0076 & 0.0068 & 0.0084 \\
\hline$\rho_{\delta}$ & $\mathrm{AR}(1)$ of sep shock & $\mathrm{B}$ & {$[0.8,0.1]$} & 0.8931 & 0.8677 & 0.9230 \\
\hline$\sigma_{\delta}$ & std of sep shock & IG & {$[0.01,0.1]$} & 0.1718 & 0.1643 & 0.1788 \\
\hline
\end{tabular}

Note: This table shows the estimation results when the model is fitted to the time series of unemployment and vacancies, without using information from search intensity. For the prior distribution types, we use "G" to denote the gamma distribution, "B" the beta distribution, and "IG" the inverse gamma distribution. 


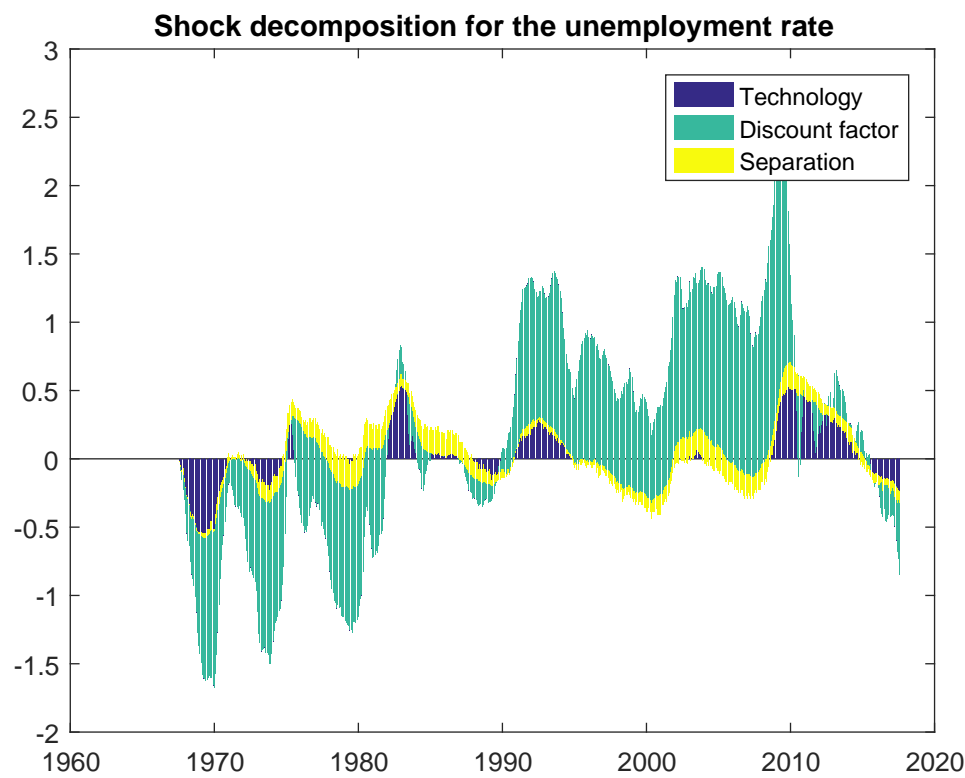

FiguRE A1. Historical decomposition for the unemployment rate. 


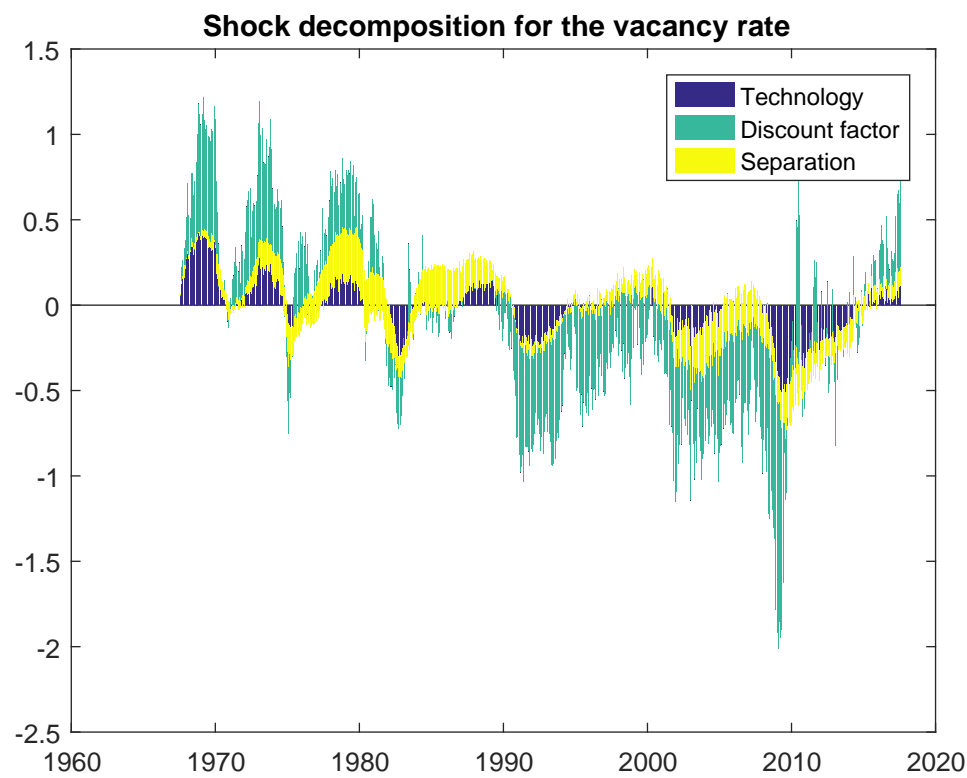

Figure A2. Historical decomposition for job vacancies. 


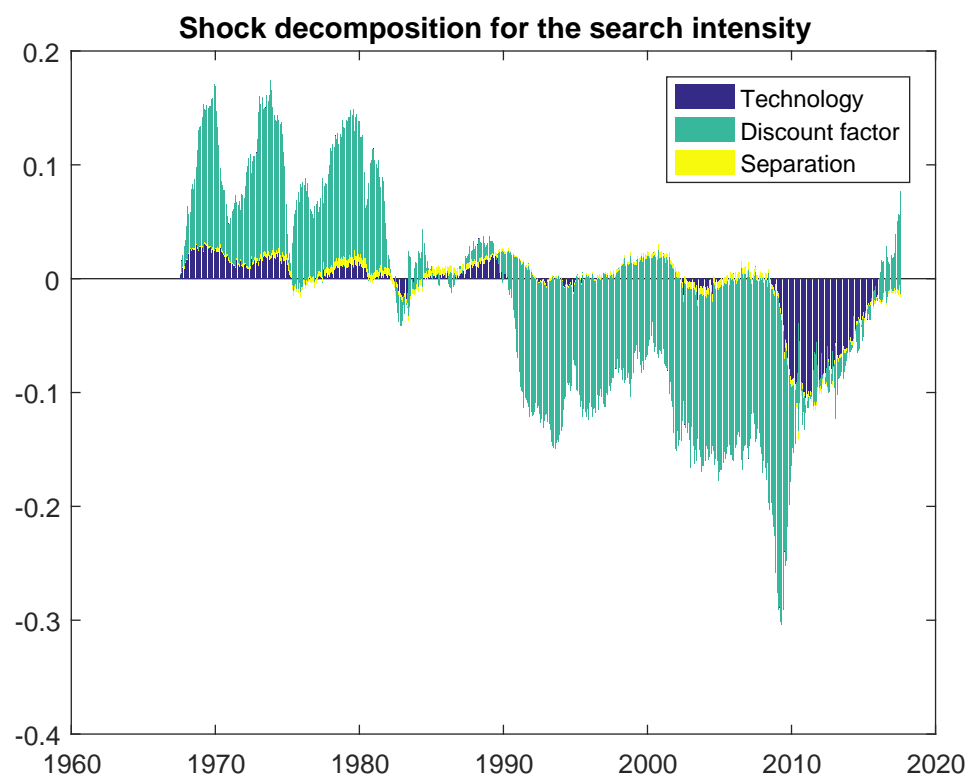

Figure A3. Historical decomposition for search intensity. 


\section{REFERENCES}

An, S. And F. Schorfheide (2007): "Bayesian Analysis of DSGE Models," Econometric Reviews, 26, 113-172.

Faust, J. And A. Gupta (2012): "Posterior Predictive Analysis for Evaluating DSGE Models," National Bureau of Economic Research Working Paper No. 17906.

Leduc, S. And Z. Liu (2019): "The Weak Job Recovery in a Macro Model of Search and Recruiting Intensity," American Economic Journal: Macroeconomics.

Shimer, R. (2005): "The Cyclical Behavior of Equilibrium Unemployment and Vacancies," American Economic Review, 95, 25-49. 